\title{
Genetix
}

Take the lead

\section{Rapid, fail-early identification of toxic compounds in secondary screening}

\author{
Screening potential therapeutic compounds for adverse biological effects has become a critical step in \\ the drug-discovery process. The biological efficacy and specificity of compounds are assessed within \\ secondary screening, and it has become desirable to also screen for adverse biological effects at this \\ early stage in the development program. CellReporter is a'fail-early' or 'early attrition' technology that \\ brings decision-making forward, delivering considerable time and cost savings in screening.
}

The CellReporter platform facilitates toxicity screening by providing a complete solution: cells can be cultured in Genetix microwell plates, treated with compounds and then assayed using Genetix' optimized cell-based assays, the Astral Assays. Plates are then imaged on the CellReporter using specific software tailored to each assay, generating an easy-to-interpret assessment of, for example, cell viability. The system images cells at up to six fluorescence wavelengths and combines this information with brightfield images to illustrate cellular morphology. Microwell plates are imaged rapidly: an entire 384-well plate is imaged at two fluorescence wavelengths, including autofocus, in under 10 minutes.

\section{The CellReporter gain}

The crux of the system is fail-early screening. This is achieved by a rapid, proprietary auto-focusing method that enables on-the-fly imaging and concomitant image analysis. Toxicity studies are an essential component of progressing compounds through the drug-discovery process. Molecules must be screened for nonspecific, adverse biological properties such as cytotoxicity. As a substantial number of compounds fail during absorption, distribution, metabolism, excretion and toxicity (ADMETox) studies because of their toxicity, it is of paramount importance to have an effective early indication of any compounds that show cytotoxic effects. CellReporter combines rapid imaging and analysis with ease of use and optimized assays to facilitate the early identification of aberrant compounds.

\section{Screening of cytotoxic compounds}

The Astral Viability Assay combines staining by a cytoplasmic, livecell dye that is detectable using a FITC filter, with staining by an allcell dye-which binds DNA and thus labels the nuclei of all cells-

\section{Janice D Broadbridge, Anna Swan \& Nicol D Watson}

Genetix Ltd. Queensway, New Milton BH25 5NN, UK. Correspondence should be addressed to N.D.W. (nicol.watson@genetix.com). detectable using a DAPI filter. The all-cell nuclear dye can be used to normalize for the number of cells. The cytoplasmic dye readily enters live cells, where active cytoplasmic esterases cleave it, producing a highly fluorescent, well-retained dye that has little effect on intracellular processes. Dying and dead cells do not retain the fluorescent dye because of compromised cell membranes.

The Astral Cytotoxicity Assay takes a new approach to assessing cytotoxicity in cell-based assays. Uniquely, the assay combines a dye (detectable with a DAPI filter) that labels the nuclei of all cells with labeling by a dye (detectable with a TxRed ${ }^{\circledR}$ or $C y 3^{\text {TM }}$ filter) that stains only dead cells. The dead-cell dye is excluded from live cells because of its inherent positive charge but readily enters dead cells owing to the compromised cell membrane.

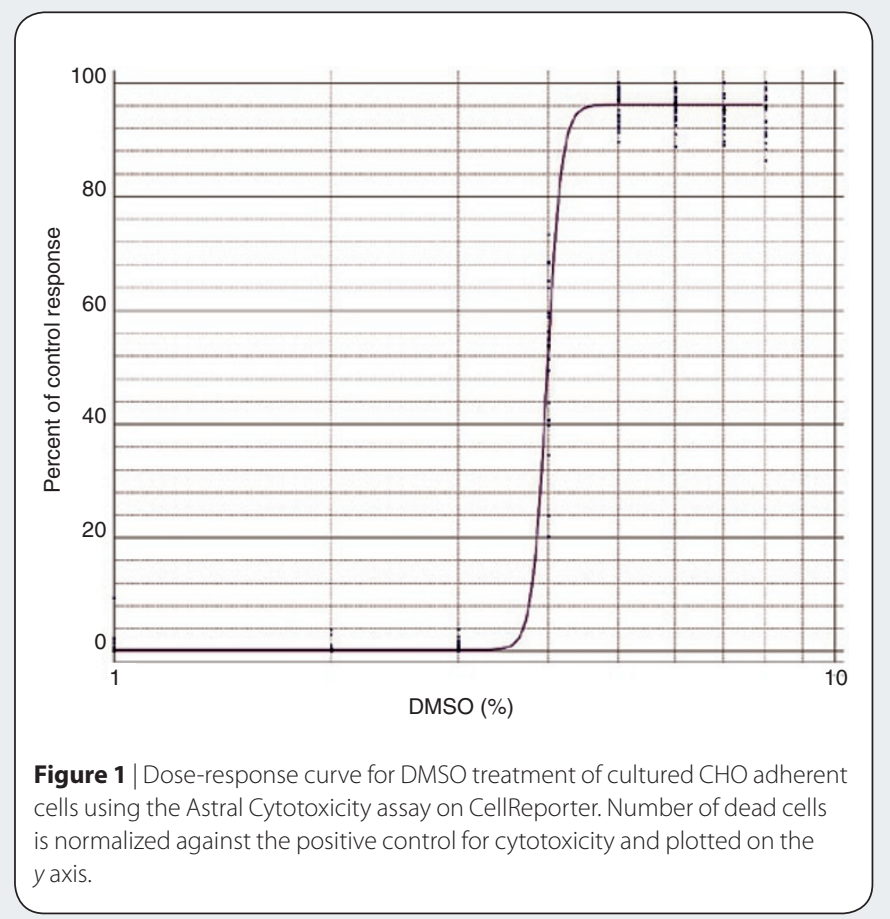


a
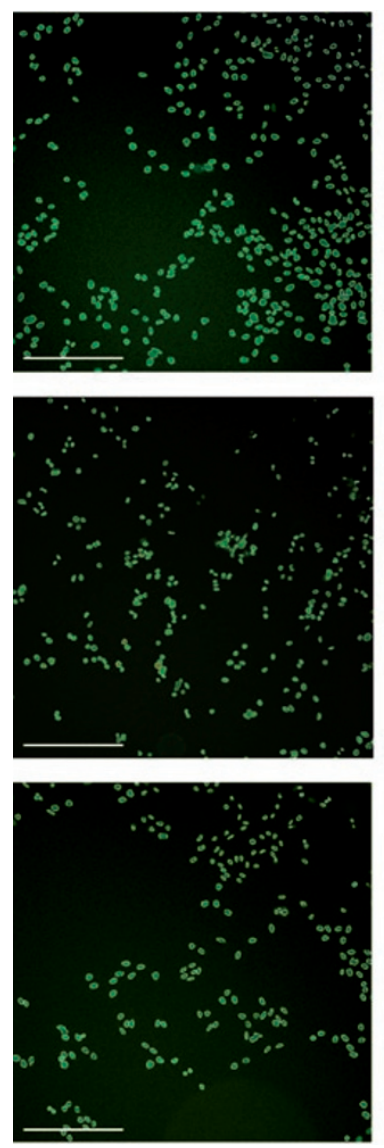

b
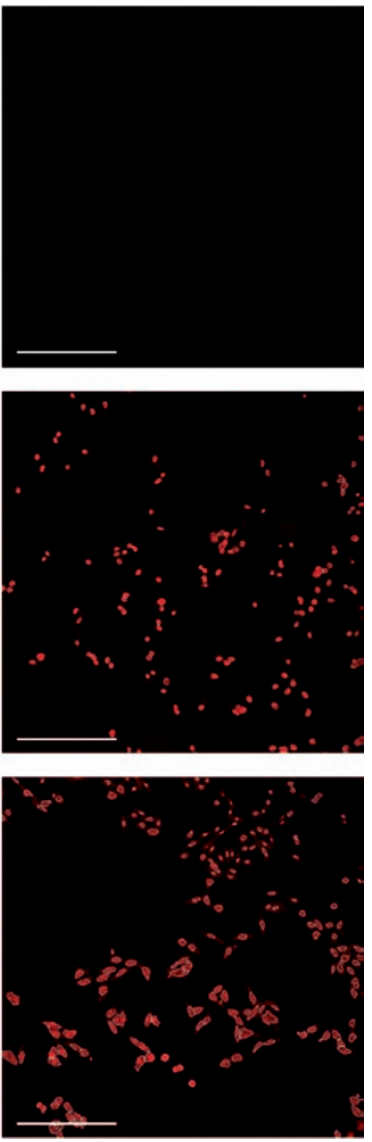

Figure $\mathbf{2}$ | Assessment of cellular viability using the Astral Cytotoxicity assay. $(\mathbf{a}, \mathbf{b})$ Colorized images of cell nuclei stained with the all-cell dye to determine cell number (a) and of dead cells stained with the dead-cell dye (b). DMSO concentration was 1\% (top), 6\% (middle) and 8\% (bottom). As DMSO concentration increased, cell viability decreased. Scale bars, $250 \mu \mathrm{m}$.

We seeded Chinese hamster ovary (CHO) cells in 384-well plates and grew them for $3 \mathrm{~d}$. To mimic exposure to a cytotoxic compound, we incubated the cells with various concentrations of DMSO for $10 \mathrm{~min}$. Then we added the Astral Viability or
Cytotoxicity Assay components to the wells and incubated the plates in the dark for 30 minutes at $37{ }^{\circ} \mathrm{C}, 5 \% \mathrm{CO}_{2}$. The plates were imaged and analyzed on CellReporter using the respective software applications. The optimized, high-throughput protocols mean that wash steps are not required. Immediately after image analysis, the CellReporter's on-board software displayed the dose-response curve for DMSO titration (Fig. 1). Crucially, respective images from each of the fluorescence channels (and brightfield images) are available for each well, enabling cell morphology and/or aberrations within the well to be visualized (Fig. 2).

We optimized the Astral Viability and Cytotoxicity assays to realize the potential of the high sensitivity of CellReporter and offer a rapid, straightforward assay that uses less reagent per well compared with other commercially available kits: the Astral Viability Assay on CellReporter uses almost half of the live-cell dye amount required for competitors' systems. Moreover, the use of only the two fluorescence channels in each assay kit enables the multiplexing of additional cellular readouts.

\section{Conclusions}

With the current drive to bolster drug pipelines, there is considerable focus on early decision-making regarding the therapeutic potential of a compound or biotherapeutic. One step in the decision-making process is the assessment of any adverse biological effect. The CellReporter platform, which combines the optimized Astral Assay kits with rapid imaging hardware and easy-to-use onboard software, facilitates a 'fail-early' approach to screen compounds or biotherapeutics for adverse biological effects, expediting decision-making and delivering considerable cost savings in bringing a drug to market.

TXRED is a trademark of Invitrogen. Cy3 is a trademark of GE Healthcare.

This article was submitted to Nature Methods by a commercial organization and has not been peer reviewed. Nature Methods takes no responsibility for the accuracy or otherwise of the information provided. 